\begin{tabular}{|l|l} 
Revista Educación \\
ISSN: 0379-7082 \\
ISSN: 2215-2644 \\
revedu@gmail.com \\
Universidad de Costa Rica \\
Costa Rica
\end{tabular}

\title{
Análisis del modelo de gestión escolar en instituciones educativas públicas multigrado de San Ignacio, Perú
}

Garcia Caucha, Humberto

Análisis del modelo de gestión escolar en instituciones educativas públicas multigrado de San Ignacio, Perú

Revista Educación, vol. 45, núm. 2, 2021

Universidad de Costa Rica, Costa Rica

Disponible en: https://www.redalyc.org/articulo.oa?id=44066178003

DOl: https://doi.org/10.15517/revedu.v45i1.40537

\section{(c) (1) 90}

Esta obra está bajo una Licencia Creative Commons Atribución-NoComercial-SinDerivar 3.0 Internacional. 


\title{
Análisis del modelo de gestión escolar en instituciones educativas públicas multigrado de San Ignacio, Perú
}

A School Management Model Analysis of Multigrade Public Schools in San Ignacio, Peru

\author{
Humberto Garcia Caucha \\ Universidad César Vallejo, Perú \\ humbeca1976@gmail.com \\ iD https://orcid.org/0000-0003-3735-7305
}

DOI: https://doi.org/10.15517/revedu.v45i1.40537

Redalyc: https://www.redalyc.org/articulo.oa?

id $=44066178003$

Recepción: 26 Marzo 2020

Aprobación: 04 Agosto 2020

\section{Resumen:}

El presente estudio denominado, "Análisis del modelo de gestión escolar en instituciones educativas públicas multigrado de San Ignacio", cuyo objetivo es analizar el modelo de gestión escolar actual de las escuelas públicas multigrado de la provincia de San Ignacio, región Cajamarca, Perú, se enmarca dentro del paradigma de la Nueva Gestión Pública y el enfoque por procesos. El tipo de investigación es no experimental, diseño descriptivo-propositivo, desarrollado sobre una población de 298 personas directoras de escuela pública multigrado, entre ellos y ellas se escogió una muestra de 58, quienes colaboraron en el desarrollo de los instrumentos de acopio de información. Se consideró la gestión escolar como exclusiva variable de estudio, con cuatro dimensiones: administrativa, pedagógica, institucional y comunitaria. Para identificar el nivel de calidad de la gestión escolar, la información requerida se consiguió mediante la técnica de la encuesta y como instrumento el cuestionario, esta información se analizó a través del software estadístico SPSS, interpretados en figuras y tablas. Los resultados conseguidos establecieron que la dimensión de gestión administrativa alcanzó 31\% para el nivel regular y 69\% para el nivel bueno; mientras que la dimensión de gestión pedagógica alcanzó 58.6\% para el nivel deficiente, y 41.4\% para el regular. La dimensión de gestión institucional, por su parte, alcanzó el 100\% para el nivel regular; asimismo la dimensión de gestión comunitaria, un 75.9\% para el nivel regular; y un $24.1 \%$, para el nivel bueno. A manera de conclusión, la dimensión más tratada es la gestión administrativa con $69 \%$ en el nivel bueno; y la que requiere mayor trabajo es la dimensión gestión pedagógica, al reportar un $58.6 \%$ en nivel deficiente. En el marco de los resultados de este estudio, las autoridades del Ministerio de Educación deben promover políticas educativas que ayuden a mejorar la calidad de la educación en el área rural, centradas al cierre de brechas en cuanto a la gestión escolar de las instituciones públicas multigrado.

Palabras ClaVe: Análisis, Modelo, Gestión escolar, Instituciones educativas multigrado.

\section{Abstract:}

The objective of this study is to analyze the current school management model for multigrade public schools in San Ignacio, located in Peru's Cajamarca Department and based on the country's process-focused New Public Management model. Research was based on a non-experimental design with a descriptive-purposeful focus conducted among 298 multigrade public school principals, of which 58 were selected as a sample group, helping to devise the data collection forms. The only designated study variable was school management with branched out into four dimensions: administrative, pedagogical, institutional and community. School management quality was assessed through a survey questionnaire and the results analyzed using SPSS Statistical Software and interpreted in graphs and tables. An average level of Administrative Management was gauged at 31\% and a good level at $69 \%$. Pedagogical Management was considered to be poor at $58 \%$ and average at $41.4 \%$. Institutional management was considered to be average at $100 \%$ and Community Management was average at $75.9 \%$ and good at $24.1 \%$. The study concludes Administrative Management had received the most attention with $69 \%$ as a good level, whereas Pedagogical Management was considered as a poor performer at $58.6 \%$. Based on the results, it is recommended that the Ministry of Education promote educational policies to, specifically, focus on improving Education in rural areas with the goal of closing existing learning gaps among multigrade public schools.

KEYWORDS: Analysis, Model, School Management, Multigrade Schools. 


\section{INTRODUCCIÓN}

La Organización de las Naciones Unidas para la Educación, la Ciencia y la Cultura [UNESCO] (2013) informa que, a partir del año 2000, en América Latina se experimentó un importante desarrollo de la economía, que permitió implementar nuevas políticas en favor de la educación; sin embargo, la desigualdad económica de la población, la no reducción de la pobreza y altas tasas demográficas en zonas rurales, constituyen limitaciones para desarrollar una educación de calidad en las instituciones educativas públicas rurales. A esto se suma el insignificante aumento del gasto público en educación, que en promedio es del 4,5\% al 5,2\% del PBI, el cual se ve reflejado en la cobertura escolar, más no en la calidad. Con esto se logra un mínimo progreso en el cierre de brechas de la calidad educativa en ámbitos rurales.

En este contexto, el constante cambio de reformas educativas y el nivel centralizado en la formulación de políticas en países como Ecuador, Perú y República Dominicana han limitado a las personas directoras de instituciones educativas a definir sus propias políticas educativas (OREALC/UNESCO, 2014). La autoridad central es el Ministerio de Educación; en consecuencia, quienes ocupan la dirección no son protagonistas de una política educativa acorde al contexto económico, político, cultural, étnico, etc.

Líderes mundiales, a través de la Organización de las Naciones Unidas (ONU), propusieron 17 objetivos de desarrollo sostenible que buscan disminuir la pobreza, darle mayor valor al ser humano, desterrar el hambre, tener en cuenta el cambio climatológico, ofertar una educación inclusiva y de calidad, con equidad, que dé oportunidades a todos y durante toda la vida (Murillo y Duk, 2017). Resulta que, a pesar de que en uno de estos objetivos destaca la educación, todavía persisten significativas brechas de equidad y con mayor profundidad en zonas indígenas rurales y de frontera. Razones suficientes para la cooperación de todos los actores educativos en opinar, colaborar en la toma de decisiones en políticas para asegurar que todo el alumnado desarrolle sus potencialidades de manera integral.

La enseñanza en contextos rurales de Latinoamérica se imparte en las escuelas multigrado o unidocentes, que representan un alto porcentaje y se ve obstaculizada por la dualidad de funciones del directivo, tanto pedagógica como administrativa. Lo que implica sobrecargas laborales de las personas directoras, que a la vez son desarrolladas de forma paralela, invirtiendo una buena cantidad de tiempo y esfuerzo, limitando una gestión escolar de calidad (Miranda, 2018). Esta particularidad de la persona docente directora de escuelas multigrado de atender hasta tres o cuatro grados al mismo tiempo, marca la diferencia en sus funciones laborales, teniendo que responder a los aspectos de gestión administrativa, pedagógica, institucional y comunitaria, evidenciando que la inclinación en su mayoría es hacia la gestión administrativa.

El Instituto Nacional de Estadística e Informática (INEI) indica que, del total de instituciones educativas públicas del Perú, el 14,8\% es de nivel secundario; el 47,0\%, de primaria; y el 38,2\%, inicial. De los porcentajes indicados, en la zona urbana, el mayor porcentaje está en inicial, con el $42,8 \%$; y el mayor porcentaje en la zona rural está el nivel primario, con el 54,3\%. Asimismo, se indica que el 64,5\% de los tres niveles educativos está en el área rural. En el nivel primario, según número de docentes, las instituciones educativas pueden clasificarse: unidocentes, polidocentes, multigrado o polidocente completo. A nivel del país, el 40,2\% de las instituciones educativas primarias son polidocentes multigrado; y están en segundo orden, con 35,3\%, las unidocentes. (INEI, 2018).

Por otro lado, el Ministerio de Educación del Perú [MINEDU] (2019) considera que en el área rural la cantidad de estudiantes por aula en instituciones educativas públicas rurales, corresponde para instituciones unidocentes a 15 estudiantes; para instituciones multigrado, 20 estudiantes y para instituciones polidicentes, 25 estudiantes. Esta realidad peruana acerca de la educación rural permite que a la persona directora de escuela multigrado se le asigne aula a cargo y atienda a estudiantes de grados diferentes, que a la vez realice trámites documentarios, atienda a padres de familia, recepcione visitas de autoridades, participe en reuniones de trabajo convocadas por instancias de gestión superiores, etc. Lo que implica, sobrecarga laboral en desmedro de centrar su atención en actividades pedagógicas en el aula. 
En este sentido, implementar cambios, desde la mirada de la Gestión Pública en la zona rural, en el sector educación, requiere asumir compromisos consensuados entre los actores educativos y agentes sociales de las diferentes tramas sociales. En el Perú, décadas atrás, la política educativa estaba regentada por los gobiernos de turno, de acuerdo con sus propios intereses. En la actualidad, ha pasado a ser política de Estado, apostando a lograr una educación de calidad e inclusiva, a incrementar la asignación presupuestal del sector, teniendo como ejes el mejoramiento de la infraestructura y el desempeño docente con el propósito de incrementar niveles de aprendizaje de los estudiantes de educación básica regular (Guadalupe, León y Rodríguez, 2017).

A pesar de haber logrado avances significativos en la implementación de estas políticas educativas, hasta la fecha no se demuestran los resultados esperados en las últimas evaluaciones estandarizadas como PISA 2018, donde el Perú ocupa uno de los más bajos resultados (The Organisation for Economic Co-operation and Development [OECD], 2019). Esto hace prioritario retomar la discusión acerca de una política educativa que guíe y ordene las prioridades del sector educación, apostando por un modelo de desarrollo para el país con equidad e inclusión como política de Estado que quizá no se encuadra en las apuestas de la OECD, para seguir disminuyendo las brechas entre las zonas más vulnerables: rurales, de frontera, campesinas, indígenas, etc.; involucrando a la sociedad civil a nivel de territorio, dado que el éxito de la política se efectiviza en el ámbito de aplicación, mas no desde el escritorio.

El MINEDU (2018b) ha centrado su mirada en la educación rural, tal es el caso que ha publicado el D.S No 013-2018-MINEDU,donde se aprueba la política educativa de atención a ámbitos rurales, demostrando interés a nivel nacional por mejorar el servicio educativo a los estudiantes de áreas rurales para que desarrollen sus competencias integralmente, en equidad de condiciones con los estudiantes de la ciudad.

La población rural en el Perú representa el $24 \%$ de la población total, con más de 6,9 millones de personas. En economía, los pobladores del ámbito rural son favorecidos con políticas de desarrollo, indicando que el $43.8 \%$ es clasificado como pobre y un $13.2 \%$ es clasificado como pobre extremo; en tanto que la zona urbana está representada por un $13.0 \%$ y $0.9 \%$ en sus respectivas categorías. En el sector educación, los datos estadísticos indican significativas diferencias entre ámbitos geográficos: en el sector rural, el promedio de escolaridad es de 8 años, por debajo de los 12 años de la zona urbana. Similar situación se da por género: mujeres entre 25 a 34 años tienen un año menos de estudios $(7,2)$ en relación con los hombres $(8,7)$; también una menor tasa de secundaria concluida (47.8\%) en jóvenes de 17 a 18 años contra un 48,1\% de hombres. Mediante estos indicadores es posible determinar ciertas brechas, a las que la política de atención educativa debe prestar atención (Instituto Nacional de Estadística e Informática (INEI, 2018).

Buscando fortalecer la gestión escolar desde la óptica de la Gestión Pública, el MINEDU (2018a), con la R. M. N ${ }^{\circ}$ 526-2018, tiene como propósito mejorar la educación rural creando el cargo de Dirección de Red Educativa Rural, cuya finalidad es potenciar el trabajo en las redes educativas rurales, dando prioridad a la gestión pedagógica que ayude a mejorar logros de aprendizaje en los estudiantes.

Para cumplir con el propósito de esta investigación se planteó el siguiente objetivo general: analizar el modelo de gestión escolar Vigente, en las instituciones educativas públicas multigrado de la provincia de San Ignacio.

Este objetivo general se logró desarrollando los siguientes objetivos específicos: Diagnosticar el nivel de gestión escolar en instituciones educativas públicas multigrado de la provincia de San Ignacio, a través de aplicación de una encuesta a personas directoras de estas instituciones.

Determinar los factores que limitan el cumplimiento de la gestión escolar en las instituciones educativas públicas de la provincia de San Ignacio.

Diseñar una propuesta de gestión escolar para las Instituciones educativas públicas multigrado de la provincia de San Ignacio.

Validar la propuesta de gestión escolar para las instituciones educativas públicas multigrado de la provincia de San Ignacio, mediante juicio de expertos. 


\section{Antecedentes}

De los estudios identificados y analizados sobre gestión escolar, merecen resaltar los siguientes aportes:

Quintana (2018) resalta la importancia de delimitar la concepción teórica de gestión escolar para alcanzar el cometido, que solo se alcanza cuando está íntimamente relacionada con la realidad educativa y su fundamento pedagógico. Sugiere ver las capacidades que poseen las instituciones educativas como recursos humanos, bienes y materiales según el entorno sociocultural y el respeto a sus costumbres, tradiciones, conocimientos y aspiraciones.

Por su parte, Maita (2018) considera que la labor docente es una actividad sacrificada en el sector público, pero, al mismo tiempo, es indispensable para alcanzar el desarrollo de los pueblos, además de contribuir con la preparación de los aprendizajes y la gestión articulada con la comunidad e identidad y profesionalidad docente. El aporte invita a valorar las variables de gestión educativa, desempeño docente y cultura organizativa como parte de un todo.

Asimismo, Del Aguila (2019), en su Tesis Doctoral, busca a través del Modelo JEC aplicado por la UGEL Lamas aplicar una propuesta estratégica. Formula una serie de preguntas que son aplicadas a las personas usuarias de la UGEL Lamas y los resultados que se obtienen de la gestión de las direcciones de las JEC; obteniendo como respuesta que la calidad de la Gestión Educativa Estratégica es relativamente baja, en donde se percibe que el factor tiempo es la que tiene prioridad.

Finalmente, Abanto, Pérez y Neciosup (2019) en su trabajo de investigación, aplicado en un instituto de EBR del distrito de San Pablo, orientado a contribuir con la mejora de la calidad del sistema educativa desde la EBR, concluyen que la gestión escolar se ubica en la categoría eficiente, debido los diferentes actores que califican como eficiente, con el $90,9 \%$ en las y los directores, con el $89,7 \%$ en las y los profesores, con el $76,9 \%$ en las y los alumnos y solo el $55,2 \%$ en las y los progenitores y/o apoderados/as. Por lo tanto, el estudio analizado permite visibilizar que la Gestión Escolar en todas sus dimensiones va a depender de la dirección de quien lidera las prácticas educativas dentro de su establecimiento.

\section{MARCo TEÓRICo}

La Gestión Pública es un conjunto de organizaciones, acciones y procesos que el estado implementa a través de políticas nacionales enfocadas en la adecuada utilización de recursos públicos para satisfacer las múltiples necesidades de los ciudadanos con eficiencia y eficacia e impeler el desarrollo nacional (Weber, 1984). Para Weber, en la administración del estado es esencial un modelo burocrático para ejercer el poder público, sobre la base de los conceptos de calidad, eficiencia y racionalidad, que se caracteriza por cargos definidos, jerarquías estandarizadas de autoridad y responsabilidad, personal competente para las funciones otorgadas, normas que rigen los actos, la permanencia en el puesto con posibilidad de ascender. Sin embargo, este modelo weberiano limita los servicios estatales que cada ciudadano debe percibir de manera oportuna y personalizada acorde a sus expectativas.

En contraposición al modelo burocrático weberiano, emerge la Nueva Gestión Pública (NGP) como un modelo postburocrático (Barzelay, 2003). Este enfoque orienta la gestión pública por resultados, validados por los ciudadanos, el mismo se entiende en términos de producción, lo cual implica delegar facultades en la toma de decisiones a las y los trabajadores; un aspecto importante del post burocratismo y las relaciones humanas en el trabajo.

Del mismo modo, Lima (2016) expone un modelo postburocrático de Gestión Pública más innovador, extrapolado de la gestión privada, donde la transigencia, la responsabilidad y satisfacción del usuario son anagramas de este modelo.

Con los lentes de la Gestión Pública se busca mejorar la toma de decisiones de las autoridades a favor del sector público; desde este enfoque de análisis, observar hacia la parte interna de la organización implica 
reconocer en qué subsistemas se sitúa el problema. Y si la mirada es hacia la parte externa, será hacia las acciones que implementan las políticas públicas, los proyectos, planes o programas (Arbulú, 2018). Otro aspecto importante desde el punto de vista de la Gestión Pública es asociar la asignación de recursos presupuestales a bienes y servicios y a resultados. Estos resultados deben permitirse medir en logros y en bienestar de la población (Soplapuco, 2020). Contrastando este enfoque presupuesto por resultados, la gestión escolar en instituciones educativas públicas multigrado debe medirse en relación con los logros de aprendizaje de los estudiantes.

En el marco de los desafíos de mejora de la gestión escolar, el Ministerio de Educación del Perú, dentro de su política de trabajo, pone énfasis en las tareas pedagógicas. Es por ello que se tiene que concebir la gestión escolar como un sistema con engranajes que deben funcionar de manera sincronizada (MINEDU, 2014).

El estudio de la gestión escolar se aborda teóricamente en un marco filosófico, axiológico, epistemológico y antropológico, atendiendo planteamientos de la antropología filosófica, la teoría de las organizaciones educativas, además del enfoque de gestión que sincronice todas sus acciones. La antropología filosófica demanda tener presente que las acciones que configuran la gestión escolar involucran personas que piensan en su destino y cada día contribuyen con sus pensamientos para mejorarlo. Por consiguiente, las acciones en una institución educativa deben responder a reflexiones constantes del hombre: acerca de su origen, su existencia, y por qué está sobre la tierra. (Beuchot, 2019).

De otro lado, la tendencia más destacada respecto a la organización como componente fundamental de la gestión escolar exige considerar el estilo de liderazgo pedagógico frente a la diversidad de posiciones sobre la educación y las bases de la gestión escolar (Bolívar, 2010).

En cuanto a la perspectiva epistemológica de la gestión escolar, siguiendo lo propuesto por García y Rojas (2003), el docente directivo se ubica en un espacio desde el cual juzga y toma decisiones sobre los aspectos de gestión administrativa, pedagógica, institucional y comunitaria, y estas decisiones varían según la percepción que tenga de la realidad como punto de partida, preguntándose: ¿qué medios utilizar para cambiar la realidad de la gestión escolar en mi institución educativa?, ¿qué conceptos manejar para enfrentar con mayor eficiencia los cambios a implementar en la gestión escolar?

No puede obviarse el hecho de que la Gestión Pública y la Ética están estrechamente ligadas; en cuanto la ética es un pilar articulador, asumiendo el papel que integre la Gestión Pública, sin olvidar que una buena Gestión Pública estará fortalecida con la actuación de la decencia de los funcionarios públicos (Buruto y Ganga, 2012).

En las últimas décadas, el concepto de NGP se muestra como tendencia y ha sido puesto en agenda educativa, dado que el sector educación administra cantidades importantes de presupuesto y de trabajadores, por lo que está predispuesto a constantes reformas educativas. Este enfoque ha modificado la gestión escolar en las instituciones educativas, orientada por principios como: gestión basada en resultados, rendición de cuentas y la autonomía (Verger y Normand, 2015).

En el marco de lo propuesto por Walsh (2005), Perú en la actualidad proclama la diversidad cultural como una posibilidad de desarrollo en el ámbito cultural, económico, político, etc., por lo que la gestión escolar en las escuelas rurales debe tener como eje principal el enfoque intercultural.

Asumiendo el concepto de gestión en la escuela que plantea la UNESCO (2011) con enfoque sistémico, para este estudio, por la naturaleza de las acciones, se consideran las siguientes dimensiones: administrativa, pedagógica, institucional y comunitaria. 


\section{MÉTODo}

\section{Tipo de estudio y diseño de investigación}

\section{Tipo de investigación}

El estudio "Modelo de gestión escolar en las instituciones educativas públicas multigrado de la provincia de San Ignacio" (Tamayo 2003) es una investigación que se encuadra dentro del paradigma positivista, método cuantitativo, tipo de investigación descriptiva, porque tiene en cuenta las características en su análisis e interpretación de la gestión escolar, además se trabajó sobre realidades de hechos ocurridos en las escuelas multigrado de la provincia de San Ignacio, donde se interpretaron los datos obtenidos.

Con este estudio se buscó describir las características, identificar e interpretar las percepciones de las y los representantes legales de las escuelas multigrado Públicas de la provincia de San Ignacio sobre el nivel de gestión escolar, considerando las siguientes dimensiones: dimensión administrativa, pedagógica, institucional y comunitaria, que se vienen desarrollando desde la década anterior.

\section{Diseño de la investigación}

En esta investigación se asume un diseño descriptivo que consiste en un proceso ordenado en la averiguación de información que se requiere para medir el nivel de gestión escolar en las instituciones educativas públicas multigrado de San Ignacio, teniendo como sustento los fundamentos teóricos y, según los resultados obtenidos, analizar los puntos de quiebre y proponer un nuevo modelo de gestión escolar (Hurtado, 2012).

Además, el estudio asumió la metodología de complementariedad, dado que no se toman decisiones de manera unilateral; al contrario, se llega a consensos por razones que favorecen a la mayoría. Corresponde a un enfoque mismo mixto con prevalencia del enfoque cuantitativo, por lo que los resultados serán representados en tablas y figuras estadísticas, y en resúmenes (Hurtado, 2012).

El diseño seleccionado asume la Figura 1, donde se expone el diseño trabajado por el investigador:

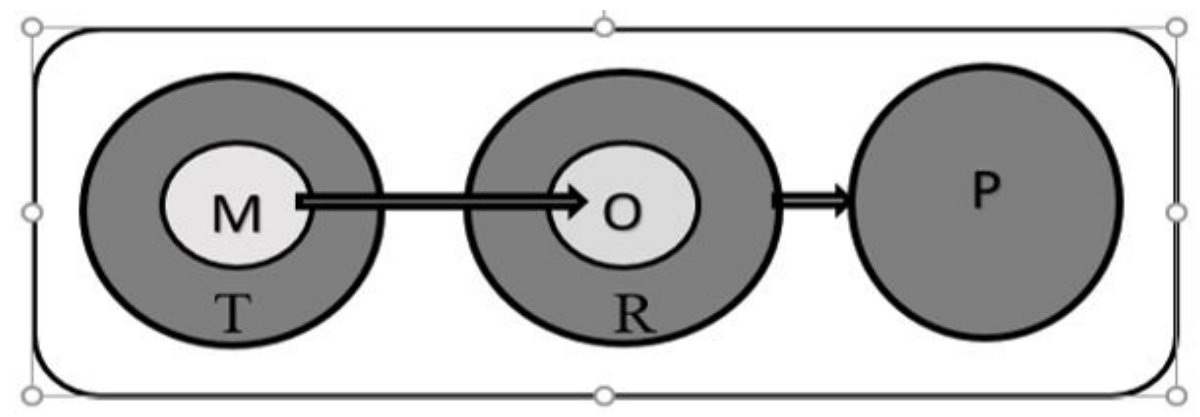

FIGURA 1

Diseño de Investigación

Fuente: Elaboración propia

Donde:

$\mathrm{M}=$ Modelo

$\mathrm{T}=$ Información teórica sobre la variable

$\mathrm{O}=$ Objeto de estudio

$\mathrm{R}=$ Realidad observada

$\mathrm{P}=$ Propuesta 


\section{Población, muestra y muestreo}

Población. En la presente investigación se consideró a todas las personas directoras de las instituciones educativas públicas multigrado del ámbito de UGEL San Ignacio. Todo trabajo de investigación tiene que estar representado por un conjunto de personas u objetos que se desea estudiar (López, 2004).

\section{TABLA 1}

Población estimada de entre directores y directoras de las instituciones educativas públicas multigrado de la provincia de San Ignacio

$\begin{array}{lll}\text { Distritos } & \text { Cantidad/docentes } & \text { Porcentaje } \\ \text { San José de Lourdes } & 48 & 16 \\ \text { San Ignacio } & 69 & 23 \\ \text { La Coipa } & 49 & 17 \\ \text { Huarango } & 34 & 11 \\ \text { Namballe } & 26 & 9 \\ \text { Tabaconas } & 45 & 15 \\ \text { Chirinos } & 27 & 9 \\ \text { TOTAL } & 298 & 100 \%\end{array}$

Fuente: Estadistica de la calidad Educativa [ESCALE], (2019)

En la Tabla 1, se detalla la población entre directoras y directores de instituciones educativas públicas multigrado de San Ignacio consideradas para esta investigación.

Muestra. La muestra estuvo representada por una parte de la población de todas las personas directoras de los distritos de la provincia de San Ignacio. Tomando como referencia a Hernández, Fernández y Batista (2014), quienes precisan que la muestra es parte de la población y representa a sus características principales.

En conclusión, la muestra fue de 58 directores y directoras de instituciones educativas públicas multigrado y se obtuvo aplicando la fórmula de población finita:

$$
\begin{aligned}
& n=\frac{N x \mathrm{Z}_{a}^{2} x p x q}{d^{2} x(\mathrm{~N}-1)+\mathrm{Z}_{a}^{2} p x q} \\
& n=58,44 \text { Directores }
\end{aligned}
$$

La muestra encuentra su sustento técnico en la fórmula de Fischer y Navarro (1996), quienes teniendo en cuenta el teorema del límite central ubicado entre $\mathrm{N} \geq 30$ y $\mathrm{N} \leq 100$; el autor considera pertinente trabajar con la fórmula de estos autores porque la muestra siempre va a ubicarse en el rango de 30 a 100 sujetos cuando la población es medianamente pequeña; pero si la población fuera 100 , se trabajaría con la misma población considerada como población muestral y no se aplicaría fórmula alguna, donde:

$\mathrm{n}=$ Tamaño de la muestra

$\mathrm{N}=$ Tamaño de la población o universo

$\mathrm{Z}=$ Parámetro estadístico que depende de la $\mathrm{N}$

e $=$ Error de estimación máximo aceptado

$\mathrm{p}=$ Probabilidad de que ocurra el evento

$\mathrm{q}=(1-\mathrm{p})$ Probabilidad de que no ocurra el evento

Muestreo

Se partió de la definición de Hernández y Carpio (2019), y se aplicó un muestreo probabilístico, considerando cualquier elemento que forme parte del universo de la población que se escogió como parte de la muestra del total de las personas directoras de instituciones educativas multigrado de la UGEL de San Ignacio de la región Cajamarca, hasta completar el tamaño calculado de 58 direcciones. 


\section{Técnicas e instrumentos de recolección de datos, validez y confiabilidad}

Durante el “Análisis del modelo de gestión escolar en escuelas multigrado de San Ignacio", la técnica utilizada fue la encuesta, y el instrumento un cuestionario, herramienta que permitió obtener información fidedigna sobre el nivel de gestión escolar en escuelas multigrado en sus dimensiones: administrativa, pedagógica, institucional y comunitaria (Ynoub, 2011).

De la misma manera se aplicó una entrevista a las y los especialistas de la UGEL San Ignacio, que está construida a través de preguntas abiertas sobre asistencia técnica en gestión escolar que brinda la UGEL a las personas directoras y la que éstos reciben del Ministerio de Educación.

\section{Validez del instrumento}

El cuestionario orientado a medir la gestión escolar en instituciones educativas públicas multigrado de San Ignacio, fue validado por tres personas profesionales conocedoras del tema y con grado de maestría y doctorado.

\section{Confiabilidad del instrumento}

Siguiendo a Cruz, Olivares y González (2014), la persona responsable de la investigación, a través del Software estadístico SPSS, comprueba la variable de estudio o experimenta al realizar una observación controlada. Por ende, dota del nivel de confiabilidad al instrumento de evaluación en un trabajo de investigación, dándole consistencia y coherencia, porque sus resultados no solo son válidos para una realidad, sino que se extrapolan a otras realidades similares. El puntaje obtenido corresponde a un nivel alto de confiabilidad superior a 0.800 .

\section{Procedimiento}

Se validó el cuestionario a través de tres personas expertas, y fue aplicado a la muestra equivalente a 58 personas directoras de las escuelas multigrado de la jurisdicción de la provincia de San Ignacio, después se procesaron e interpretaron los datos utilizando el software estadístico SPSS; también se elaboraron resúmenes de las entrevistas realizadas a las personas especialistas de la UGEL, luego se procedió a elaborar el modelo de gestión escolar, para poner en marcha el modelo a través de Redes Educativas.

\section{Método de análisis de datos}

Para Niño (2011), el método de análisis de datos "implica un proceso mental complejo, el cual comprende varias operaciones (de alguna manera ya mencionadas) como descomponer, examinar, reconocer, sintetizar, conceptualizar, relacionar, explicar y comprender" (p. 105).

Los instrumentos fueron analizados a través a del software estadístico SPSS para encontrar la confiabilidad del instrumento con el método estadístico alfa de Cronbach, y el Excel para el procesamiento de la estadística descriptiva simple.

La información recogida en las entrevistas se sistematizó en base a un análisis categorial: categorías y subcategorías.

\section{Aspectos éticos}

Para Acevedo (2018), el aspecto ético en una investigación busca el beneficio con los resultados y los experimentos de todas las personas que están involucrados en la investigación, de forma individual o colectiva.

Se cumplieron los principios éticos asumidos por la Universidad César Vallejo respecto al consentimiento informado de los sujetos de la investigación y la respectiva autorización de la UGEL de San Ignacio. 


\section{Resultados}

Objetivo específico $\mathbf{N}^{\circ}$ 01. Diagnosticar el nivel de gestión escolar en instituciones educativas públicas multigrado de la provincia de San Ignacio.

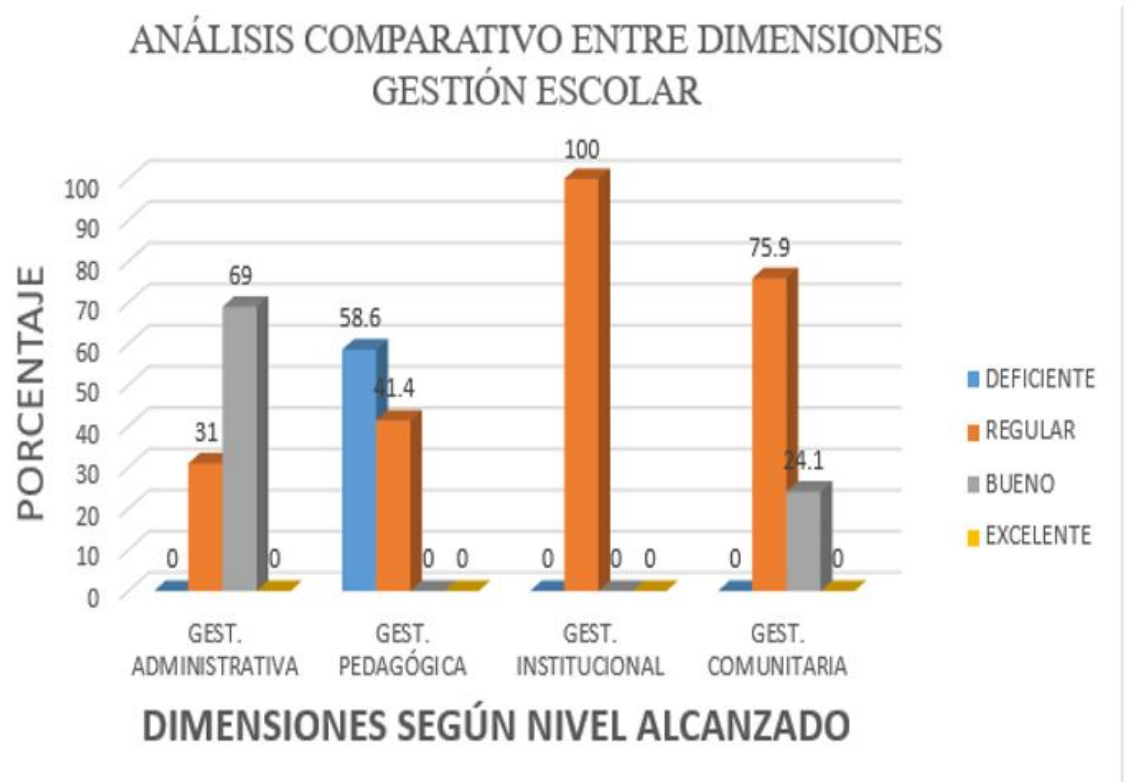

\section{FIGURA 2}

Nivel de gestión escolar según dimensiones

Fuente: Elaboración propia

En la Figura 2, según las direcciones encuestadas, se evidencia que la dimensión que requiere mayor atención es gestión pedagógica, al reportar un 58.6\% de nivel deficiente, seguido de la dimensión gestión institucional con el 100\% de nivel regular; muy de cerca se ubica la dimensión gestión comunitaria, con un $75.9 \%$ de nivel regular, dejando constancia que la dimensión que está más trabajada es la gestión administrativa, alcanzando un $69 \%$ de nivel bueno.

Objetivo específico $\mathbf{N}^{\circ} \mathbf{0 2}$. Determinar los factores que limitan el cumplimiento de la gestión escolar en las instituciones educativas públicas de la provincia de San Ignacio.

Los factores que limitan el cumplimiento de la gestión escolar en las instituciones educativas multigrado de San Ignacio son: número elevado de instituciones multigrado, la rotación de personal que en su mayoría son contratados/as, instituciones educativas que no están organizadas en redes, el doble rol de las y los docentes (dirección y aula a cargo), interrupciones por visitas de diversas autoridades de instancias superiores, inasistencias por salud, capacitaciones o trámites. Se tendrían que remunerar las horas extra como motivación para contar con asistencia masiva en horas fuera de horario de clase.

La UGEL, para coadyuvar a la gestión escolar, brinda asistencia técnica a través de GIAS para personas directoras, visitas programadas, talleres en el periodo vacacional a instituciones educativas focalizadas. Del mismo modo, el Ministerio de Educación brinda asistencia técnica en gestión escolar a través de GIAS, tutoriales, normas técnicas y talleres.

Además, la UGEL implementa estrategias como escritorio limpio, Plataforma virtual, Trámite documentario los días sábado, acompañamiento, talleres informativos sobre documentos de gestión y normas legales y talleres de reflexión sobre el balance de gestión escolar.

Objetivo específico $\mathrm{N}^{\circ}$ 03. Diseñar una propuesta de gestión escolar para las instituciones educativas públicas multigrado de la provincia de San Ignacio. 
Se diseñó el modelo de propuesta de gestión escolar en instituciones educativas públicas multigrados, resumida en un organizador visual que considera el enfoque sistémico: entrada, procesos y salida; en el marco de las políticas públicas educativas del sector, lineamientos de política educativa regional y local, considerando como eje central la gestión escolar implementada a través de alianzas y convenios, jornadas de capacitación y reuniones colegiadas, entre direcciones de instituciones educativas multigrado, desarrollando la autonomía, la investigación y las buenas prácticas; teniendo como soporte teórico el enfoque humanista, el enfoque intercultural, el enfoque ambiental, el enfoque de indagación, entre otros con la única finalidad de obtener buenos resultados y así disminuir las brechas en relación a las instituciones educativas de la Urbe.

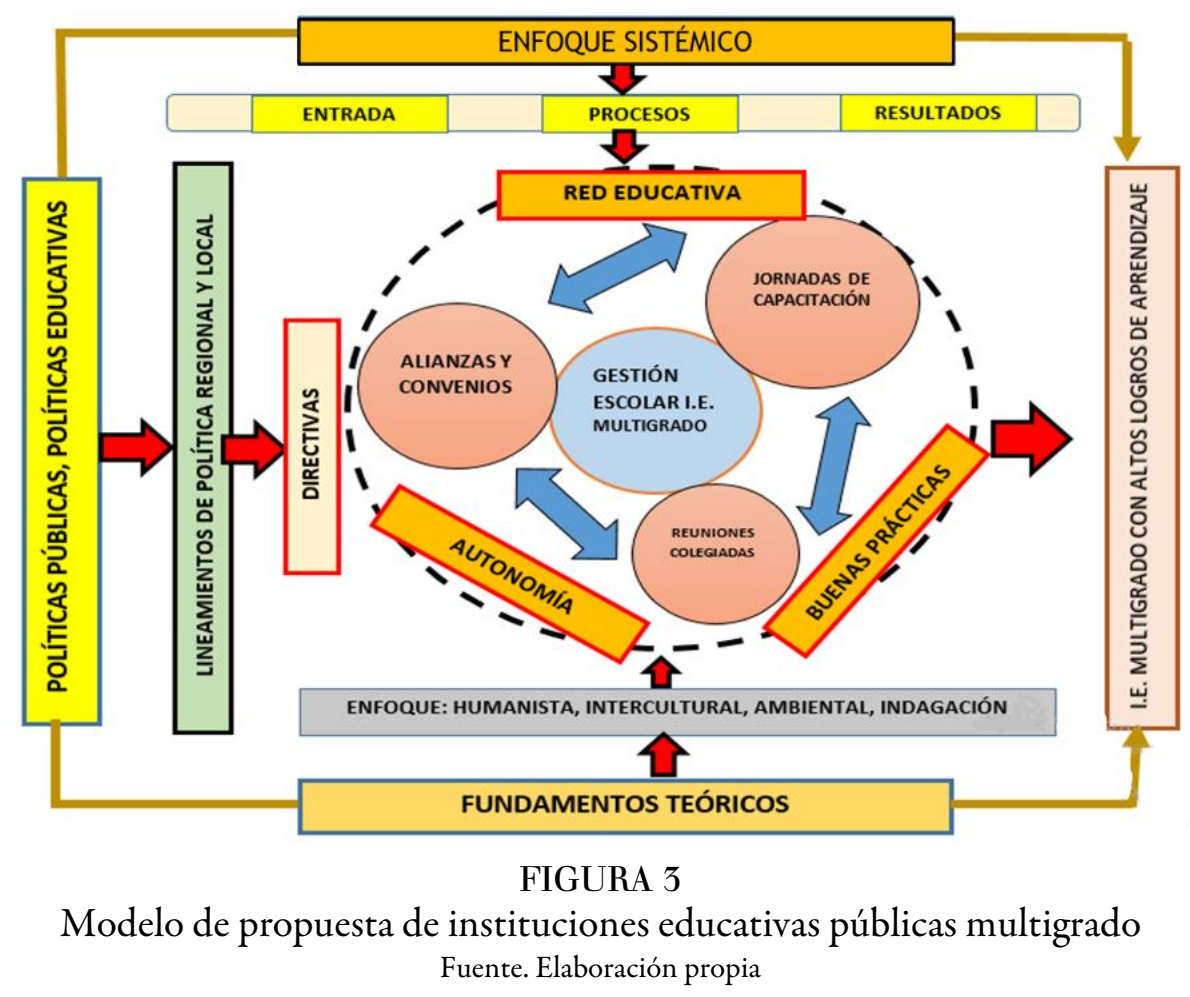

En la Figura 3, se expone el diseño del modelo de gestión escolar en instituciones educativas públicas multigrado de San Ignacio.

Objetivo específico $\mathrm{N}^{\circ}$ 04: Validar la propuesta de gestión escolar para las instituciones educativas públicas multigrado de la provincia de San Ignacio, mediante juicio de expertos.

La propuesta contó con el soporte de validación de tres personas profesionales conocedoras del tema, vinculadas la Gestión Pública en el sector educación y con experiencia como funcionarias de la UGEL San Ignacio y UGEL Lambayeque, teniendo en cuenta la redacción de los objetivos, los principios teóricos, éticomoral, la correspondencia entre la complejidad de las actividades a desarrollar y su profundidad científica, la contribución e implementación de políticas públicas de la gestión escolar, la organización y la metodología de la propuesta bajo el enfoque sistémico, propuesta o modelo de acuerdo a las pretensiones de la y/o el investigador, contribución a la Gestión Pública de la UGEL San Ignacio y su relación entre los resultados y el presupuesto asignado por el sector.

\section{Discusión}

El análisis de los resultados de la investigación debe abordarse a partir de la premisa de que la Gestión Pública en el sector educación, mantiene grandes rasgos del modelo burocrático, por lo que cuando las instancias jerárquicas inferiores asumen visiones diferentes de la gestión escolar, se producen brechas entre los conceptos 
asumidos por el Ministerio Central, las gerencias regionales y las Unidades de Gestión Educativa Locales (UGELES).

Analizar la gestión escolar en instituciones educativas públicas multigrado responde a la pregunta: ¿cuál es la situación actual del modelo de gestión escolar en las instituciones educativas públicas multigrado de la provincia de San Ignacio?, situación que condujo a observar la realidad problemática de la gestión escolar desarrollada en estas instituciones educativas ubicadas en zona rural y de frontera teniendo en cuenta sus dimensiones: gestión administrativa, gestión pedagógica, gestión institucional y gestión comunitaria.

Los resultados fueron obtenidos a través de la encuesta aplicada a las personas directoras de las instituciones educativas públicas multigrado de San Ignacio, teniendo en cuenta las dimensiones antes mencionadas, obteniendo los siguientes resultados: la dimensión que requiere mayor atención es la de gestión pedagógica, al reportar un 58.6\% de los encuestados que están en un nivel deficiente; seguida de la dimensión de gestión institucional, de la cual el $100 \%$ manifestaron que están en un nivel regular; muy de cerca se ubica la dimensión de gestión comunitaria, con un 75.9\% nivel regular, dejando constancia que la dimensión que está más trabajada es la de gestión administrativa alcanzada.

Según García y Rojas (2003), la concepción epistemológica ubica a el y la docente directiva en un espacio desde el cual juzga y toma decisiones sobre los aspectos de gestión administrativa, pedagógica, institucional y comunitaria, y estas decisiones varían según la percepción que tenga de la realidad como punto de partida. A raíz de los resultados obtenidos, se afirma que la dimensión de gestión administrativa está centrada en las tareas eminentemente de gabinete y coordinación con autoridades institucionales e interinstitucionales, generando en algunas ocasiones mayor inversión de tiempo en las personas directoras. En la actualidad, es tendencia que la dimensión de gestión pedagógica sea la más ponderada en la gestión escolar, y están bajo su responsabilidad las tareas de planificación, ejecución, evaluación de los procesos pedagógicos y didácticos, monitoreo y acompañamiento a las y los docentes, pero a la luz de los resultados obtenidos se evidencia bajo nivel de cumplimiento en esta dimensión.

Por otro lado, la dimensión de gestión institucional está relacionada con desconcentrar el poder del directivo y formar equipos de trabajo institucionales. Según los resultados obtenidos en el desarrollo de este estudio, se evidencia que esta dimensión muestra un nivel aceptable de cumplimiento.

Del mismo modo, la dimensión de gestión comunitaria está vinculada con las actividades que las y los directivos realizan con otras instituciones de los diferentes sectores sociales en pro del progreso, no solo de la institución educativa, sino del contexto social, para ello se realizan alianzas y convenios estratégicos donde ambas partes salen fortalecidas. Según los datos obtenidos en este estudio, la dimensión de gestión comunitaria evidencia un nivel bueno en relación a su cumplimiento.

El aporte de Quintana (2018) está relacionado en resaltar la importancia de la concepción teórica de la gestión escolar para alcanzar la calidad educativa, la misma que solo se logra cuando está ligada al liderazgo pedagógico. No obstante, en este estudio los resultados objetan lo mencionado por Quintana (2018), dado que la dimensión pedagógica demuestra un nivel bajo. En consecuencia, se tienen que implementar políticas para potenciar el aspecto pedagógico.

Maita (2018) valora a las variables gestión educativa, desempeño docente y cultura organizativa como parte de un todo. En ese sentido, depende de la sincronización que ejerzan cada uno de los componentes de la gestión escolar para alcanzar los objetivos propuestos.

Asimismo, el aporte que se recibe de Del Águila (2019) es el trabajo en equipo como factor valioso para el logro de un buen nivel de la gestión escolar en las Instituciones educativas públicas multigrado de la provincia de San Ignacio, corroborando de esta manera los resultados de este estudio en su dimensión comunitaria que se encuentra en un buen nivel de cumplimiento, concluyendo que el trabajo colaborativo, democrático, participativo es la base para una buena gestión escolar.

Finalmente, el aporte de Abanto et al. (2019) expresa que la gestión escolar está determinada por la forma como la dirección lidera la gestión escolar, implementando acciones de manera organizada entre pares a nivel 
territorial con participación democrática. Contrastado con los resultados de este estudio, queda demostrado que a pesar de que hay lineamientos y políticas con tendencia a fortalecer la gestión pedagógica, aún no se logran los resultados esperados.

\section{Conclusiones}

El nivel de gestión escolar de las instituciones educativas públicas multigrado de la provincia de San Ignacio registra los siguientes resultados: la dimensión gestión pedagógica 58.6\% nivel deficiente, la dimensión gestión institucional 100\% nivel regular, la dimensión gestión comunitaria $75.9 \%$ nivel regular y la dimensión gestión administrativa 69\% nivel bueno. por lo tanto, se concluye que la dimensión que requiere mayor atención la dimensión gestión pedagógica que está en un nivel deficiente como también la dimensión gestión institucional, que demuestra un nivel regular; en contraste a la dimensión gestión administrativa, que tiene un nivel bueno, y a la gestión comunitaria, que está entre un nivel regular y nivel bueno, por lo que se deduce que la persona directora de las instituciones educativas públicas multigrado de San Ignacio centra sus actividades con mayor proporción a labores administrativas, en desmedro de las actividades de aprendizaje de las y los estudiantes, que es la columna vertebral de la gestión escolar.

Son varios los factores limitantes de la gestión escolar de las instituciones educativas públicas multigrado, uno de ellos es que son un número elevado de este tipo de instituciones y están ubicadas en zonas rurales, dispersas a grandes distancias, que además no están organizadas en Redes Educativas; otro factor limitante es el doble rol del directivo y directiva: dirección y docente de aula, enfatizando el rol de dirección, debido a visitas de autoridades, monitoreo de instancias superiores, reuniones de capacitación, solución de conflictos internos y externos de personas usuarias de la institución educativa, sumándose además cada año escolar la rotación de personal que en su mayoría son contratados.

El diseño del modelo de propuesta de gestión escolar en instituciones educativas públicas multigrado de San Ignacio, se sustenta en fundamentos teóricos relacionados a la gestión escolar en escuelas multigrado bajo el enfoque humanista, intercultural, ambiental e indagación, a implementarse a través de redes educativas rurales, siendo los insumos de políticas educativas los lineamientos de política regional y local con la finalidad de obtener altos logros de aprendizaje de los estudiantes de estas instituciones educativas públicas multigrado.

El modelo de propuesta está validado con instrumentos de evaluación, que a la vez están validados por tres personas expertas con amplio conocimiento del tema y con grado de maestría y doctorado, quienes emitieron su juicio de valor en relación con la correlación de su estructura diseñada. Se recomienda a las autoridades educativas tener en cuenta los resultados de esta investigación para mejorar la gestión escolar de las instituciones educativas públicas multigrado de San Ignacio.

\section{ReFerencias Bibliográficas}

Abanto, M., Pérez, M. y Neciosup, J. (2019). School management in public educational institutions of regular basic education in the district of San Pablo - San Pablo. Sciéndo, 22(3), 187-190. doi: https://doi.org/10.17268/sci endo.2019.024

Acevedo, I. (2018). Ethical issues in cientific research. Ciencia y enfermeria, 8(1), 15-18. doi: https://doi.org/10.406 7/S0717-95532002000100003

Arbulú, C. G. (Ed.). (2018). Metodología de la Investigacion en Gestión Pública. Chiclayo, Perú.

Barzelay, M. (2003). La Nueva Gerencia Pública: invitación a un diálogo cosmopolita. Gestión y Política Pública, 12(2), 241-251 Recuperado de https://www.redalyc.org/pdf/133/13312202.pdf

Beuchot, M. (2019). Interpretación del ser humano. Barcelona, España: Herder Editorial, S.L.

Bolivar, A. (2010). El liderazgo educativo y su papel en la mejora. Una revisión actual de sus posibilidades y limitaciones. Psicoperspectivas, 9(2), 9-33. doi: https://doi.org/10.5027/psicoperspectivas-Vol9-Issue2-fulltext-112 
Buruto, J. y Ganga, F. (2012). For an Ethics of Public Management. (C. d. (CISPO), Ed.) Polis: Revista Latinoamericana (32), 1-11. Recuperado de https://journals.openedition.org/polis/6479

Cruz, C., Olivares, S. y González, M. (2014). Metodología de la investigación. México: Grupo Editorial Patria S.A.

Del Aguila, R. (2019). Propuesta de Gestión Educativa estratégica, basado en el Modelo JEC de la Unidad deGestión Educativa local Lamas - 2019. (Tesis Doctoral). Universidad Cesar Vallejo, Lamas. Recuperado de https://hd l.handle.net/20.500.12692/38481

Estadística de la Calidad Educativa [ESCALE]. (2019). Padrones de asignaciones temporales. Recuperado de http:/ /escale.minedu.gob.pe/rm093

Fischer, L. y Navarro, A. (1996). Introducción a la investigación de mercados. México: McGraw Hill Interamericana Editores S.A.

García, M. y Rojas N. (2003). Concepciones epistemológicas y enfoques educativos subyacentes en las opiniones de un grupo de docentes. Investigación y Postgrado, 18(1), 11-21. Recuperado de http://ve.scielo.org/scielo.php?sc ript $=$ sci_arttext\&pid $=$ S1316-00872003000100003

Guadalupe, C., León, J. y Rodríguez, J. (2017). Estado de la educación en el Perú: Análisis y perspectivas de la educación básica. Lima: Impresiones y Ediciones Arteta E.I.R.L. Recuperado de http://repositorio.minedu.gob.pe/handl e/MINEDU/5692

Hernández, C. y Carpio, N. (2019). Introducción a los tipos de muestreo. Alerta, Revista Cientifica Del Instituto Nacional De Salud, 2(1), 75-79. doi: https://doi.org/10.5377/alerta.v2i1.7535

Hernández, R., Fernández, C. y Batista, M. (2014). Metodología de la investigación. México: Interamericana Editores, S.A.

Hurtado, J. (2012). Metodología de la Investigación. Bogotá: Ciea - Cypal.

Instituto Nacional de Estadística e Informática [INEI]. (2018). Principales resultados de la encuesta nacional a instituciones educativas del nivel inicial, primaria y secundaria, 2018. Lima: Gráfica Burgos SAC. Recuperado de https://www.inei.gob.pe/media/MenuRecursivo/publicaciones_digitales/Est/Lib1684/libro.pdf

Lima, L. C. (2016). Evaluación Hiperburocrática. Profesorado, 20(3), 87-118. Recuperado de https://recyt.fecyt.es/i ndex.php/profesorado/article/view/54592

López, P. (2004). Población, muestra y muestreo. Punto Cero, 9(8), 69-74 Recuperado de http://www.scielo.org.bo/ scielo.php?script $=$ sci_arttext\&pid $=S 1815-02762004000100012$

Maita, R. B. (2018). Gestión educativa, desempeño docente y cultura organizacional en las Instituciones Públicas de la UGEL 07-Lima 2018. (Tesis de Doctorado). Universidad Cesar Vallejo, Lima. Recuperado de http://reposito rio.ucv.edu.pe/handle/UCV/2729

Ministerio de Educación del Perú [MINEDU]. (2019). R.V.M 307-MINEDU-2019 "Disposiciones para el proceso de racionalización en el marco de la ley de la reforma educativa magisterial en instituciones educativas públicas de educacion básica". Lima, Perú. Recuperado de shorturl.at/ijquC

Ministerio de Educación del Perú. [MINEDU]. (2014). Marco de buen desempeño directivo. Lima, Perú. Recuperado de http://repositorio.minedu.gob.pe/handle/MINEDU/5182

Ministerio de Educación del Perú. [MINEDU]. (2018a). Resolución Ministerial No 526-2018-MINEDU. Lima, Perú: Munedu: Recuperado de https://www.gob.pe/institucion/minedu/normas-legales/199161-526-2018-minedu

Ministerio de Educación del Perú. [MINEDU]. (2018b). D.S. No 013-2018-MINEDU. Lima, Perú: Minedu. Recuperado de shorturl.at/swKL6

Miranda, L. (2018). Management in Single-Teacher Schools and Dirección 1: A Challenge to Reach Educational. Educare, 22(3), 1-30. doi: https://doi.org/10.15359/ree.22-3.10

Murillo, J. y Duk, C. (2017). SDG 4 (and 16) as a goal for the next years. Revista Latinoamericana de Educación Inclusiva, 11(2), 11-13. doi: https://doi.org/10.4067/S0718-73782017000200001

Niño, V. (2011). Metodología de la investigación. Bogotá, Colombia: D'vinni S.A. 
OREALC/UNESCO. (2014). El liderazgo escolar en America Latina y el Caribe: un estado del arte en base a ocho Sistemas Escolares de la Región. Santiago: Imbunche Ediciones Ltda. Recuperado de https://unesdoc.unesco.or g/ark:/48223/pf0000232799

Quintana, Y. (2018). Education Quality and School Management: A Dynamic Relationship. Educación y Educadores, 21(2), 259-281. doi: https://doi.org/10.5294/edu.2018.21.2.5

Soplapuco, P. (febrero, 2020). Estrategias de gestión administrativa para mejorar la ejecución presupuestal del programa estratégico logros de aprendizaje del nivel primario en la UGEL de lambayeque. En II International Congress of Public Management, congreso llevado a cabo en Cesar Vallejo College. Florida, Estados Unidos.

Tamayo, M. (2003). El proceso de la investigación cientifica. México: EDITORIAL LIMUSA, S.A.

The Organisation for Economic Co-operation and Development (OECD). (2019), PISA 2018 Results (Volume I): What Students Know and Can Do. Paris: OECD Publishing. doi: https://doi.org/10.1787/5f07c754-en

UNESCO en el Perú. (2011). Manual de Gestión para Directores de Instituciones Educativas. Lima, Perú: Lance Grafico S.A.C.

UNESCO (2013). Situación Educativa de América Latina y el Caribe: Hacia la educación de calidad para todos al 2015. Santiago, Chile: Salesianos Impresores S.A. Recuperado de http://www.unesco.org/new/fileadmin/MULTIM EDIA/FIELD/Santiago/images/SITIED-espanol.pdf

Verger, A. y Normand, R. (2015). Nueva gestión pública y educación: elementos teóricos y conceptuales para el estudio de un modelo de reforma educativa global. Educação \& Sociedade, 36(132), 599-622. doi: 10.1590/ ES0101-73302015152799

Walsh, C. (2005). Inter - Culturality, Knowledge and decolonialism. Signo y Pensamiento, 24(46), 39-50. Recuperado de https://revistas.javeriana.edu.co/index.php/signoypensamiento/article/view/4663

Weber, M. (1984). Economia y sociedad. Esbozo de sociología comprensiva. México, D. F.: Fondo de Cultura Económica. Ynoub, R. (2011). El proyecto y la metodología de la investigación. Argentina: Cengage Learning Argentina.

\section{INFORMACIÓN ADICIONAL}

Cómo citar: Garcia-Caucha, H. (2021). Análisis del modelo de gestión escolar en instituciones educativas públicas multigrado de San Ignacio, Perú. Revista Educación, 45(2). Recuperado de http://doi.org/10.155 17/revedu.v45i1.40537 